\title{
Multiscale Modeling of Polymeric Materials
}

\author{
PI: Janna K. Maranas \\ Department of Chemical Engineering \\ Penn State University \\ jmaranas@psu.edu \\ 814)863-6228
}

Mathematical, Information and Computational Sciences

Early Career Principal Investigator Program

Project Start Date: August 15, 2002

\section{Final Project Report}

Review of Project Objectives: Our overall objective for the three-year duration of this project is to design an adaptive, learning, simulation technique for polymers covering the range from atoms to coarsegrained chains, and apply the technique to the description of nanofilled polymers. Accomplishing this objective requires four activities, with the first two corresponding to the first two project years, and the second two to take place in the third project year.

1. Generalize and expand on existing methods to generate coarse-grained [CG] potentials [i.e. those where a single bead represents between ten and thirty atoms] from atomistic simulation results and potentials.

2. Develop protocols for overlaying atomistic structures on coarse-grained chains.

3. Use the results from Tasks 1 and 2 to embed the two levels of modeling in a single simulation, with "communication" between the two levels.

4. Verify the dynamic multi-scale simulation on a polymer melt, and use it in initial studies of nanoparticles in a polymer matrix.

Objectives added during the course of the proposed work: As work on this project proceeded, we identified needs for obtaining accurate dynamic properties from coarse grained models. We also realized that the polymer immediately surrounding a nanoparticle filler must be described atomistically, which requires a means to join an atomistic region with a coarse-grained region within a single simulation. As a result, we added two further objectives:

5. Parameterize the coarse-grained potentials to deliver accurate dynamic properties over a large range of time scales and for segmental and whole chain mobility.

6. Devise a method appropriate to describe a spatial region in which an atomistic region of the simulation box is smoothly joined to a coarse-grained region.

Specific Tasks: The specific goals for the project support the original objectives, as stated in the proposal, as well as the additional objectives.

\section{- Develop a library of CG potentials}

- Develop/code optimization procedure to select the best CG potential from atomistic level simulation data.

- Develop the CG simulation code.

- Perform atomistic simulations to set the initial values of CG potentials.

- Develop methods to replace atomistic detail to CG coordinates.

- Test these methods by comparing directly to full atomistic simulations.

- Conduct atomistic simulations of a nanoparticle surrounded by a polymer matrix. 
- Implement embedded simulation code.

- Investigate methods for selecting subsystems for updates.

- Set nanoparticle-polymer potentials based on atomistic simulations.

- Develop CG nanoparticle-polymer potentials.

- Implement embedded simulation of nanofilled systems

- Investigate the influence of nanoparticle shape on its properties/potentials.

- Test CG potentials, and modify if necessary, to obtain accurate properties.

- Determine if CG potentials are independent of chain length

- Test CG potentials for dynamic properties within the time range of the original atomistic model.

- Test CG potentials for dynamic properties out of the time range of the original atomistic model.

- Extend existing method for joining atomistic and CG simulation regions to chain molecules.

The tasks in bold have been completed and will be described in this report. The remainder were not completed in order to accommodate the new objectives.

\section{Project Accomplishments:}

The remainder of the document describes the accomplishments of the project in detail. It is based on the following publications that have resulted or are currently in preparation from the project:

1. S. Natarajan and J.K. Maranas "Discretization Parameters in fine-grained lattice simulations of linear and branched polymers” J. Chem. Phys., 118, 9053 (2003).

2. S. Fullerton and J.K. Maranas "A molecular interpretation of dynamics in boron oxide nanoparticles", J. Chem. Phys., 121, 8562 (2004).

3. S. Fullerton and J.K. Maranas "A molecular dynamics study of the structural dependence of boron oxide nanoparticles on shape”, Nano Letters, 5, 363 (2005).

4. P. Depa and J.K. Maranas, "Speed up of dynamic observables in coarse-grained molecular dynamics simulations of unentangled polymers”, J. Chem. Phys. 123, 094901 (2005).

5. C. Chen, P. Depa, V. Garcia-Sakai, J.K. Maranas, J.W. Lynn, I. Peral, J. R.D. Copley, “A comparison of united atom, explicit atom, and coarse-grained simulation models for poly(ethylene oxide)”, $J$. Chem. Phys., 124, 234901 (2006).

6. P. Depa and J.K. Maranas "Dynamic evolution in coarse-grained molecular dynamics simulations of polyethylene melts” J. Chem. Phys., 126, 054903 (2007).

7. C. Chen, P. Depa and J.K. Maranas "A comparison of united atoms, explicit atoms, and coarsegrained simulation models for poly(methyl methacrylate)", Macromolecules, in preparation.

8. S.K. Fullerton and J.K. Maranas "The influence of nanoparticle shape on the properties of the surrounding polymer matrix" Nano Letters, in preparation.

9. P. Depa and J.K. Maranas "Dynamic properties of chain molecules using coarse-grained simulation" Nature Materials, in preparation.

10. C. Chen, P. Depa and J.K. Maranas “ The influence of PMMA entanglement on the mobility of PEO in PEO/PMMA blends" Macromolecules, in preparation.

11. P. Depa and J. K. Maranas "Merging atomistic and coarse-grained simulations of chain molecules" $J$. Chem. Phys., in preparation.

Accomplishment 1: modeling single nanoparticles - with and without a polymer matrix: Papers 2, 3, \& 8]: We have conducted simulations of nanoparticles alone, and nanoparticles surrounded by atomistic polymer, in preparation for incorporation in a large scale, coarse-grained modeling effort. Specicially, 
we simulate single boron oxide nanoparticles, and boron oxide nanoparticles surrounded by a polyethylene oxide [a linear polymer that we have coarse-grained] matrix.

While studying the particle alone for purposes of developing CG particle-polymer potentials, we discovered some exciting aspects of its mobility that are extremely relevant to the field of amorphous materials. The atoms comprising the $1.6 \mathrm{~nm}$ particle move as collective units - either a $\mathrm{BO}_{3}$ "triangle" or three such triangles arranged to form a ring, as illustrated in the position density graph to the right. In this graph, the positions of the atoms over the first $44 \mathrm{ps}$ form a dense cluster, followed by a jump to a new location and further exploration of a local "cage" there, as indicated by the blue symbols. This jumping is part of a larger scheme in which collectively moving units follow each other in discrete hops, each being pulled by the unit moving ahead of it. It differs from other amorphous materials in
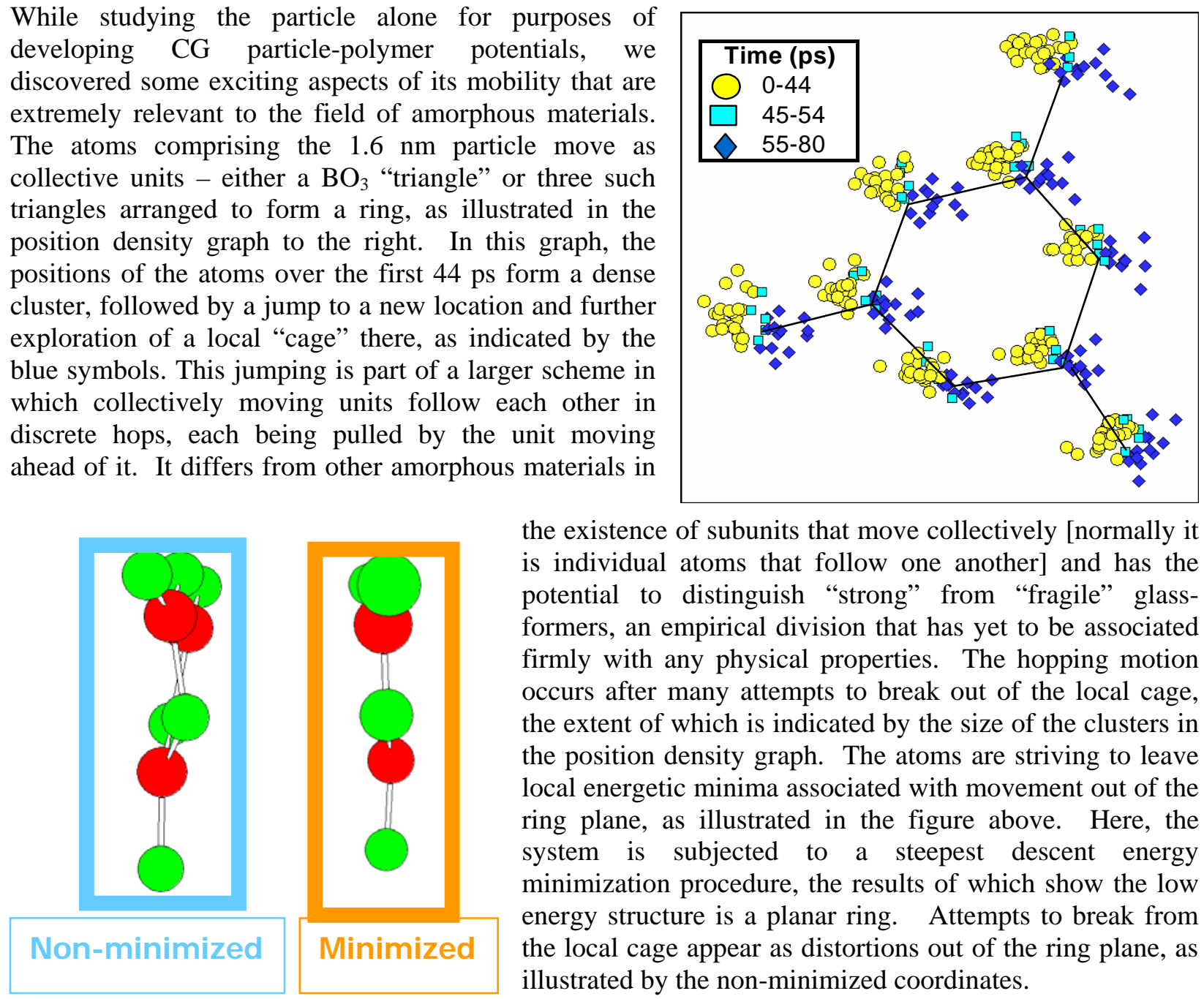

the existence of subunits that move collectively [normally it is individual atoms that follow one another] and has the potential to distinguish "strong" from "fragile" glassformers, an empirical division that has yet to be associated firmly with any physical properties. The hopping motion occurs after many attempts to break out of the local cage, the extent of which is indicated by the size of the clusters in the position density graph. The atoms are striving to leave local energetic minima associated with movement out of the ring plane, as illustrated in the figure above. Here, the system is subjected to a steepest descent energy minimization procedure, the results of which show the low energy structure is a planar ring. Attempts to break from the local cage appear as distortions out of the ring plane, as illustrated by the non-minimized coordinates.

Because of the planar ring structure in boron oxide, illustrated above, nanoparticles of small dimension could be significantly impacted by particle shape as the structure of the particle attempts to accommodate the planar ring. We have investigated this effect and found a significant difference in the internal particle structure of a spherical versus cubic nanoparticle. Specifically, whereas the spherical particle is a random network, with the rings located in the interior, in the cubic particle, the rings aggregate to the cube walls. The results in the formation of layers aligned parallel to the walls of the cube, similar to both the high density amorphous form and the low density crystalline phase of boron oxide.

Simulations of boron oxide particles, such as the one described above, surrounded by a polymer matrix have also been completed. In the simulation, we fix the positions of the atoms in the particle - they are not updated by the simulation package, but the polymer chains interact with the particle and move freely. A snapshot of the system is shown on the following page. The polymer chains pack in layers like an onion around the particle. This packing is facilitated by the oxygen atoms in the PEO, which have a preferential attraction to the particle. In addition to packing, the dynamics of the PEO chains are significantly altered by the particle. Even at the edges of the simulation box, the PEO chains move much slower than chains in a PEO melt. Shifts in structure and dynamics of PEO chains extend throughout the 
box, indicating that the influence of the particle on polymer properties has the potential of being quite long-ranged. Changes in packing are very relevant to the CG potentials, and suggest that an atomistic description must be retained near the particle surface.

Accomplishment 2: Coarse-grained simulation of a variety of polymeric systems - extra computational gain for dynamics [papers 4, 5, 6, 7, 9, \& 10]: We have developed a coarse-grained, multiscale modeling approach for long chain polymers that is able to correctly describe both structural properties and dynamic evolution. In this approach, four to six backbone atoms are grouped into a coarse-grained bead. We assign the potentials of interaction

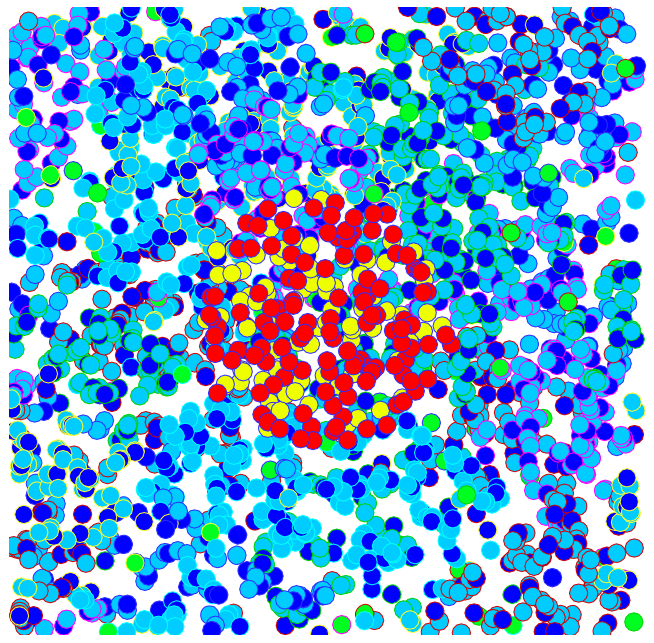
between CG beads based on an underlying atomistic simulation. As an example, consider the coarsegraining of polyethylene [PE] based on a united atom model. In this case, each CG bead consists of four united atoms. Intramolecular and intermolecular potentials are treated separately. For intramolecular potentials [CG bond stretching, bending and torsion], we determine the desired distributions of CG bond lengths, angles and torsions from the corresponding positions of united atoms. Intramolecular potentials describe different physical phenomena in the CG and UA models: for example the CG bond length encompasses two UA torsional angles. Intermolecular potentials describe the same physical phenomena in both models: the closest approach of non-bonded chain segments, or the thickness of the chain. In this case, we simply require that the intermolecular $\mathrm{g}(\mathrm{r})$ be coincident between the two models. This requires a change in the parameters used to describe non-bonded interactions: in the case of the Lennard-Jones potential, $\varepsilon$ and $r$ will take different values in the CG model. Specifically, the potential well in the CG model is shallower and displaced to larger distances.

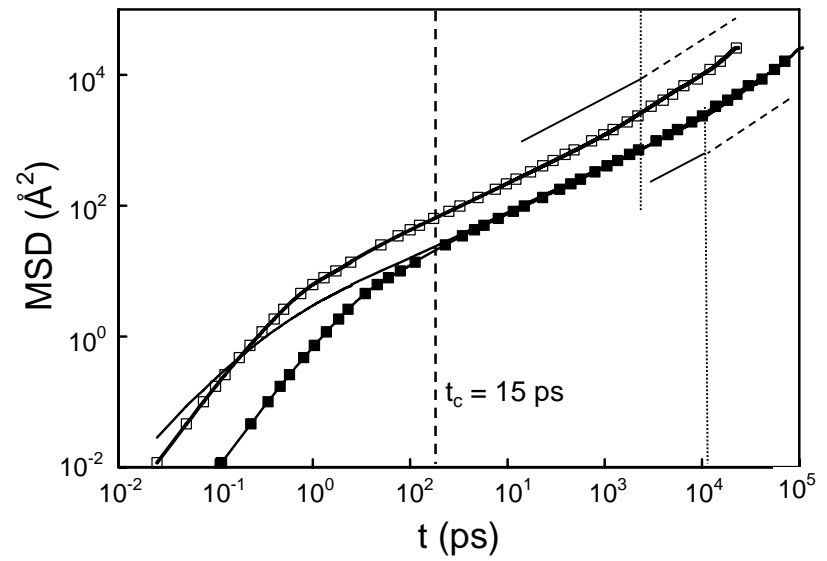

Figure 1: Comparison of monomer mean-squared displacements, for the UA model [filled symbols], the original CG model [open symbols], and the CG model with time shifted as predicted using the hyperdynamics framework [line].
Coarse-grained models are known to yield incorrect dynamic evolution. Specifically, they are too fast. We have linked this increase in dynamics to changes in the intermolecular potential described above. This has two important impacts: the difference is a constant, meaning that it can be used as further computational gain, and the difference can be predicted based on the atomistic and CG intermolecular potentials. The prediction is based on the hyperdynamics method, which is used to purposely accelerate MD simulations by "boosting" the potential of interaction. In our case, we have not purposely set out to accelerate the simulations, rather it is a result of the "softer" potentials required to attain correct structural properties in the CG model. The result is that the CG time step used in the simulation,

$\Delta \mathrm{tCG}$, is related to the actual elapsed time by $\Delta \mathrm{t}=\alpha \Delta \mathrm{tCG}$, where $\alpha$ is predicted by the hyperdynamics framework. Plotted in Figure 1 are the mean squared displacements [msd] computed from the UA and CG models, and those resulting from scaling the CG time step with the predicted value. Following this 
scaling, the msd of CG model is quantitatively accurate at times greater than a cross-over time, tc. This accuracy continues into the diffusive regime, the start of which is indicated by the dotted line.

This scaling is remarkably robust. It is independent of chain length, and accurately describes any dynamic property. As an example, in Figure 2 the diffusion coefficient determined from the CG simulations, independent UA simulations [not those used to set the potentials], and experiment are compared as a function of chain length. The agreement is excellent, even into the entangled regime. It is not immediately apparent that this would be the case: the intermolecular interactions and their time scale of association, which lead to chain entanglement, are reduced by the softer potential. However, further analysis of entangled dynamics [reptation] using the CG model provides tube diameters [37 A] consistent with experimental data. Apparently the scaling parameter predicted from physical arguments limited to the first neighbor shell has continued applicability to

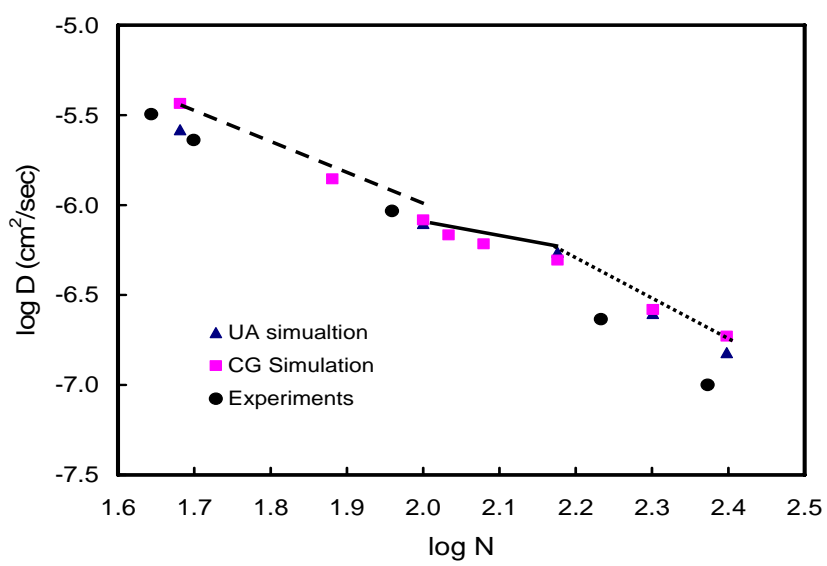

Figure 2: The CG diffusion coefficient of PE at $423 \mathrm{~K}$ as a function of chain length. Dashed, solid and dotted lines represents the short-chain, rouse, and reptation regimes respectively. Diffusion coefficients from UA simulation and experiments are also shown.

more complicated phenomena. Apart from providing accurate time evolution, there is an additional bonus, as the computational gain in shifting to a CG description is amplified by the time scaling, which we have termed the "indirect speed-up". The indirect speed-up in the case of PE is moderate [ 5], but in systems with long range interactions, it can be substantial: $\alpha=53$ for poly(ethylene oxide) and $\alpha=550$ for poly(methyl-methacrylate).

This method has several advantages: it retains connection to an underlying physical model, its force field is similar to that used for atomistic simulations and thus can be implemented in existing codes with excellent parallelization capabilities, and its dynamic evolution may be validated with experimental measurements. Because the CG model retains connection to an underlying atomistic simulation, we were able to develop methods to replace the missing atoms at any time. This allows for a multiscale simulation, where different sections of the sample under consideration are described with different levels of modeling within the same simulation. Replacing missing atoms is also useful to provide information

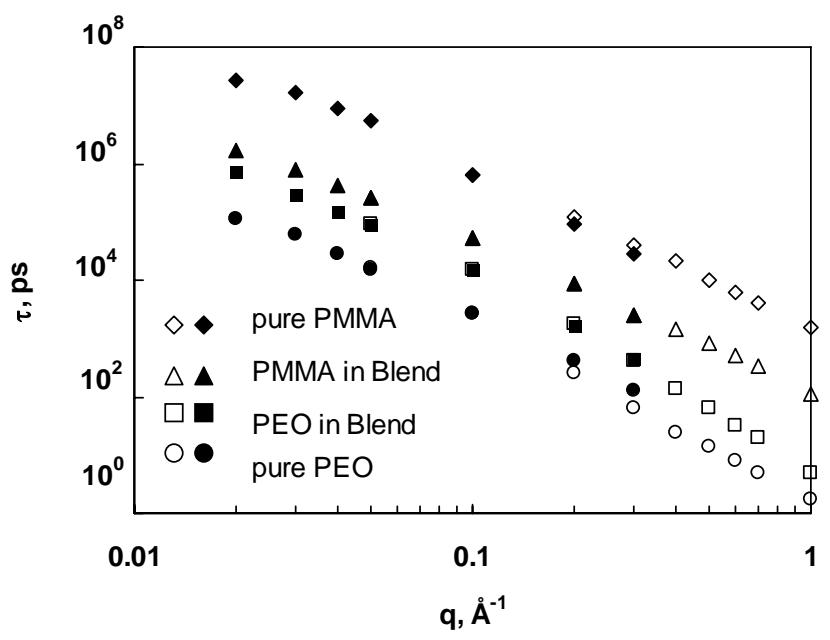

for short time and small length scale phemonema that are missing from the CG model. Before proceeding with assignment of a CG force field, the dynamics of the atomistic model are validated using dynamic neutron scattering experiments, which are performed within our group. We thus know at the outset that the dynamic evolution captured by the CG model is reliable and accurate.

We have considered four polymer melts: PE, PEO [poly ethylene oxide], PEP [poly ethylene propene] and PMMA [poly methyl methacrylate]. The latter has a large side group which must be represented in the coarse grain description. As a result, we extended our methodology such that 
coarse-grained potentials for materials with CG side chains can be specified. We have also used our approach to correctly describe structure and dynamics of polymer mixtures, in which case a number of new issues are introduced. For example, we found that the pure component CG parameters can be successfully used in mixtures, the indirect speed up of each component in a mixture is different from that in the pure components, and each component requires a different indirect speed-up for comparison to experimental or other data. When these features are implemented, the CG approach for mixtures is extremely successful, as illustrated in the Figure above, where relaxation times for both the CG and UA levels of modeling are shown for pure PEO, pure PMMA, and each component in their mixture.

Accomplishment 3: Merging of atomistic and coarse-grained simulation regions for chain molecules [paper 11]: Because we have discovered that the region immediately surrounding a nanoparticle filler is critically impacted at an atomistic level, we have implemented a method for modeling "hand-shake" zones, defined as a physical area of the simulation box where the level of description moves smoothly from atomistic to coarse-grained. Our approach is based on prior work with small molecules, in which the forces are smoothly transferred from an atomistic to a coarse-grained description within a hydrid region to avoid the large forces that would result from an abrupt transition. The idea is illustrated in the schematic simulation box to the right. A nanoparticle occupies the center of the box, immediately surrounded by a region of atomistic description, followed by the hybrid region and finally the coarse-grained region. In the original implementation, the CG particles did not have intramolecular bonding; extending this approach to chain molecules introduces CG bonded interactions, and requires dealing with two additional issues: CG

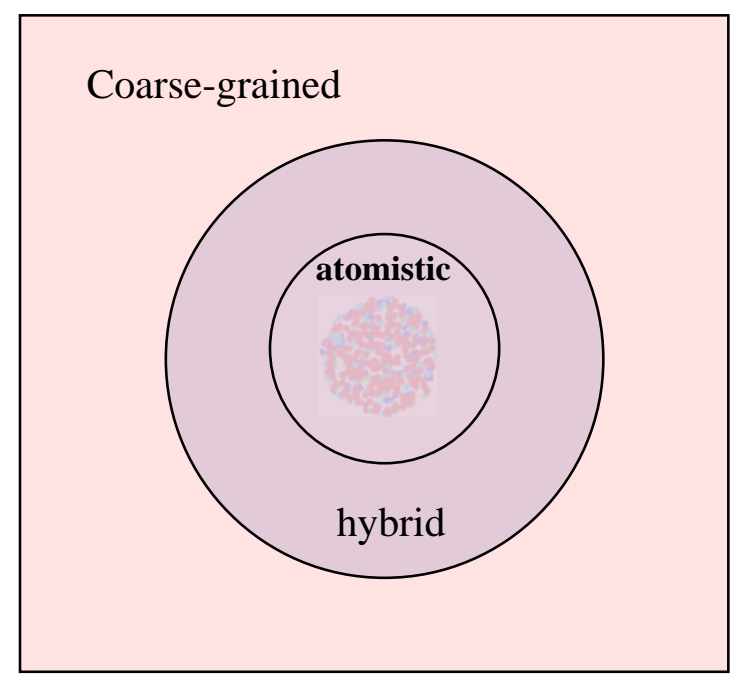
bonds or bends that cross a boundary, and ensuring that CG bonded forces vary smoothly between all regions within the simulation box. The latter was necessary for non-bonded CG forces, which were scaled using the smoothing factor $\omega$, which varies from zero to one as a function of particle position: it is zero at the coarse-grained/hybrid interface, and unity at the hybrid/atomistic interface.

$$
F_{i j}=(1-\omega) F_{i j}^{C G}+\omega F_{i j}^{U A}
$$

We introduced a similar approach for the bonded interactions. The resulting multiscale simulation maintains system properties, as illustrated by the plots below. On the left, we plot the normalized density, which remains constant as the various parts of the simulation box are probed. On the right, we show the intermolecular pair distribution function, as compared between a fully atomistic [UA], fully coarsegrained $[\mathrm{CG}]$ and the multiscale simulation.
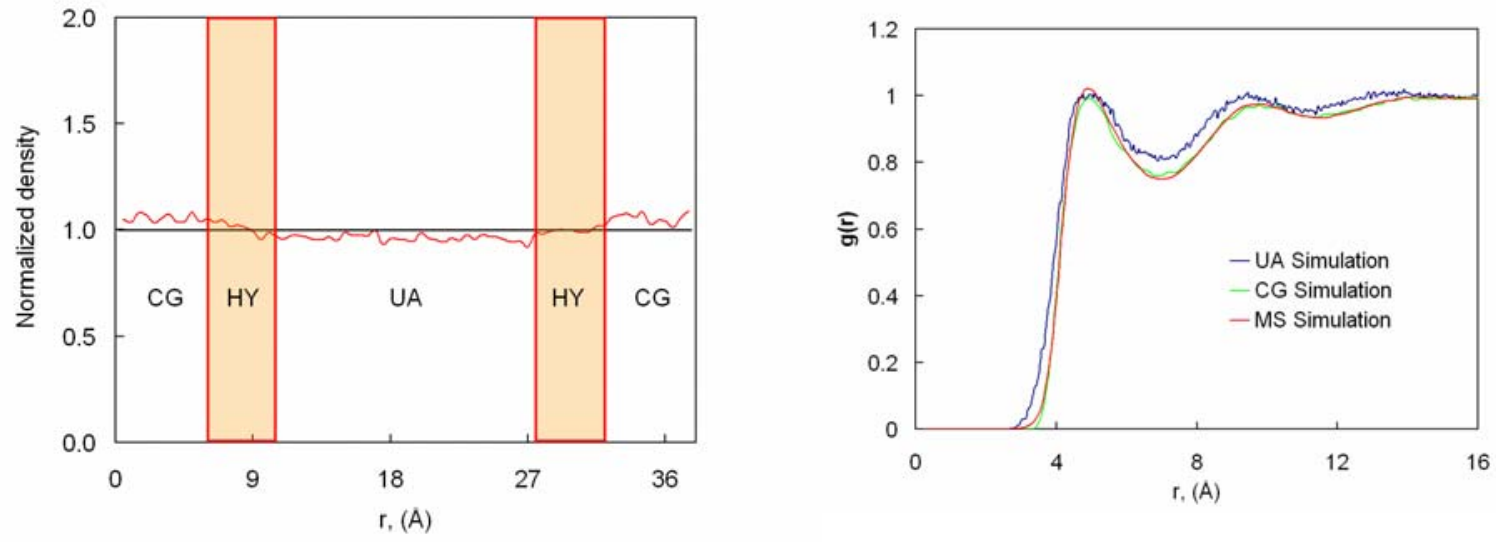